\title{
Network Performance Comparison of Light Fidelity and Wireless Fidelity
}

\author{
S.N. Arinze, G.N. Onoh and D.O. Abonyi \\ Corresponding Author: $S$. N Arinze
}

Light Fidelity is a wireless technology that uses light for data communication. It is a bidirectional network that delivers data at high speed. Mobile users can experience high data throughput with Light fidelity than the existing wireless fidelity technology. This paper mainly focuses on the network performance of Light fidelity and wireless fidelity when many devices connect to the system. The aim was carried out by simulating the wireless fidelity system in the OPNET Modeller environment. The result obtained showed that as the number of users increases, there is a reduction in data throughput and increment in latency and packet loss. Simulation of Light fidelity done in the Simulink environment showed that there were high data throughput, low latency, and low packet loss. Light Fidelity network was not significantly affected by the number of users. The result of both light fidelity and wireless Fidelity was compared using a python program. Observation showed that the network performance of the light fidelity system was better than the wireless fidelity system. The percentage improvement of the data throughput of light fidelity to wireless fidelity was 53\% while that of latency and packet loss is $39.1 \%$ and $8.78 \%$ respectively.

Index Terms - Light Fidelity, Wireless Fidelity, Throughput, Latency, Packet loss, Opnet Modeller, Simulink

\section{INTRODUCTION}

Presently, the limited bandwidth capacity of the radio frequency is not capable enough to carry numerous signal loads and deliver a successful message at higher speeds. It is a result of a large number of devices that demand higher bandwidth. When many devices connect to Wireless Fidelity (Wi-Fi) spot, the slow speed of the network leads to long processing hours. Sometimes pages will not load, videos will buffer, and email crawls to a halt. This situation usually frustrates users, especially when they struggle to process a large amount of data. Many customers tend to abandon a page if it has not loaded, thereby making them unable to download relevant documents. Many hours can be lost waiting for pages to load. One cannot get enough bandwidth that allows for enough on-peak and off-peak data and so cannot have an increased throughput. Without a proper solution to this problem, access to information and resources is challenging to get, the information will be outdated before it gets to people, many businesses will dwindle, and there will be a slowdown in social communication and networking. In response to this problem, Light Fidelity (Li-Fi) communications are the link between future data throughput and wireless data communication. The concept of Li-Fi is to transmit data using light, a receiver that captures the signal and processes the information at a

S.N. Arinze ,G.N. Onoh, D.O. Abonyi and are with Electrical and Electronic Engineering Department, Enugu State University of Science and Technology (e-mail: arinze.ndidi@esut.edu.ng, greg.nwachukwu@esut.ng, abonyi.dorathy@esut.ng.edu). 
data transfer speed higher than Wi-Fi speed (10Mbps) thereby maximizing the bandwidth and increasing throughput in a local area network.

In October 2011, companies and industrial groups in the United States of America formed the Li-Fi consortium to promote high speed optical wireless systems thereby utilizing another electromagnetic spectrum to overcome the available limited Radio Frequency (RF) spectrum. The consortium believed that more than 10Gbps achieved will download a high definition film in 30 seconds. Quite several companies offered uni-directional visible light communication products but are not like Li-Fi that is bi-directional. Data rate over 1Gbits was demonstrated using a single color LED on August 2013. Reports in October 2013 showed that Chinese manufacturers were working on Li-Fi development kits [1]. Li-Fi offers thousands of more pathways for data to travel, which means it provides unprecedented data and bandwidth [2]. It can decrease the data traffic bottlenecks. It does not penetrate the wall, thereby providing greater security from hackers. Light fidelity resolves the issue of availability, scalability, and interference. It finds its applicability where radio waves are prohibited such as airplanes, hospitals, and some research facilities

\section{Wireless Fidelity}

\section{THEORY}

Wireless fidelity is a communication technology that allows an electronic device to transfer data wirelessly using radio bands. Its main target is to get everyone connected with the internet. Wi-Fi devices are portable and based on IEEE 802.11 standards. Wi-Fi standards were developed to accommodate the growing need for faster data and improved performance of the wireless network. Though the standards differ in terms of frequency, transmission ranges, and speed but can make use of infrastructure or ad hoc network as well the same security protocols. Wi-Fi standard technologies [3] are

- Wi-Fi 802.11: it specifies three physical layer technologies, namely infrared operating at $1 \mathrm{Mbps}$, Frequency-hopping spread spectrum (FHSS) operating at $1 \mathrm{Mbits}$ or $2 \mathrm{Mbits}$ and Direct Sequence Spread Spectrum (DSSS) working at $1 \mathrm{Mbits} / 2 \mathrm{Mbits}$. It is obsolete and no longer deployed.

- Wi-Fi 802.11a: it defines the format and structure of which Wi-Fi routers and antennas transmit radio signals. It goes as fast as $54 \mathrm{Mbps}$ in the $5 \mathrm{GHz}$ band. $802.11 \mathrm{a}$ employs the Orthogonal Frequency Division Multiplexing (OFDM) encoding scheme, supports the multimedia application, produces more significant throughput, experiences less interference, has difficulty in penetrating walls and smaller wavelengths. It is presently rarely used. Its overall range or coverage is less. More access points co-locate in $802.11 \mathrm{a}$, but they are incompatible with $802.11 \mathrm{~b}$,

- Wi-Fi 802.11b: This supports the $2.4 \mathrm{GHz}$ frequency spectrum with a bandwidth of $11 \mathrm{Mbps}$. Its signal range is suitable for home appliances. $802.11 \mathrm{~b}$ is cost-effective, stable but has security issues and is affected by interference from other products. It uses only the Direct Sequence Spread Spectrum (DSSS). Devices operating in this standard are Bluetooth devices, Cordless telephone, baby monitor, microwave oven and amateur radio equipment. It has a limited number of APs, and interference is a disadvantage of this technology.

- Wi-Fi 802.11g: It uses 2.4GHz frequency for a more excellent range and supports a bandwidth of up to 54Mbps. 802.11g uses DSSS and OFDM. Its speed is fast. It is compatible with $802.11 \mathrm{~b}$, costly, flexible, limited to one AP and has interference from other $2.4 \mathrm{GHz}$ devices. An example of an $802.11 \mathrm{~g}$ device is a wireless keyboard.

- Wi-Fi 802.11n: It supports between $300 \mathrm{Mbps}$ to 600Mbps bandwidth, operates at $2.4 \mathrm{GHz} / 5.0 \mathrm{GHz}$ and was designed to improve on $802.11 \mathrm{~g}$. It has a better range, 
provides services up to $15 \mathrm{~m}$ for indoors and $457 \mathrm{~m}$ for outdoors and compatible with $802.11 \mathrm{~b} / \mathrm{g}$. It does not make use of one wireless signal and antenna but multiples of them.

- Wi-Fi 802.11i: The primary purpose of its creation was to counter security issues of Wired Equivalent Privacy (WEP). It uses two encryption methods, namely Temporal key integrity protocol, and Advanced encryption standard but cryptographers confirmed it not to be reliable.

More standards created are 802.11ah, 802.11ad, 802.11aj, 802.11ac, 802.11ax, 802.11ay, $802.11 \mathrm{az}$ but some are not yet approved. They are developed to provide long-range communications, high performance, power control and improve security issues [4]

Wi-Fi technology is unsecured though its security is configured by turning on the wired equivalent privacy (WEP) to avoid an intruder getting connected to one's wireless router and using the data for downloading movies, games and carrying out terrorist activities. Some of the advantages of Wi-Fi include easy setup and configuration, portability and no cabling. No technology is perfect. Hence it possesses its limitations which are inefficiency in longdistance data transfer, harmful to human health due to its radiation in a low gigahertz frequency, expensive implementation, compatibility and interoperability, low bandwidth and throughput, unsecured and interference from other signals.

Areas of applications of Wireless Fidelity are internet, research, conference room, mobile app, business applications, automotive segment, computerized and home applications.

\section{Light Fidelity}

Light Fidelity is a visible light communication where light waves are used as a medium to carry high-speed data. It offers broadband connectivity by using LED as signal transmitters and photodiodes as the receiving element. Li-Fi uses quick pulses of light to transmit information. Since LED can be switched ON and OFF, the optical output varies at high speed through fast and slight variations of the light-emitting diode to transmit high data. The modulation is so rapid that the human eye cannot detect it. Light Fidelity has a speed of more than 1Gbps; no limitations on capacity have not been utilized or exploited and reduce energy consumption. It can operate with existing infrastructures. LEDs used in Li-Fi are due to their brightness, low power consumption, longer lifetime, smaller size, ease dimming, high tolerance to humidity, and ability to toggle within a few microseconds.

Li-Fi uses IEEE 802.15.7 standard. The standard includes both the physical layer and the media access control layer [5]. Management issues such as addressing, acknowledgment, and collision avoidance are taken care of by MAC. Physical layers are sub-divided into three types namely

i. PHY 1: This layer is for outdoor applications with data rates in the range of $12 \mathrm{Kbit} / \mathrm{s}$ to $267 \mathrm{Kbit} / \mathrm{s}$. It recognizes the On-Off Keying (OOK) modulation.

ii. PHY 2: This layer is for indoor applications with data rates from $1.25 \mathrm{Mbit} / \mathrm{s}$ to 96Mbit/s. OOK and Pulse Position Modulation (PPM) are the modulation techniques recognized in this layer

iii. $\quad$ PHY 3: It deals with Color Shift Keying (CSK) modulation and delivers data rates from $12 \mathrm{Mbits} / \mathrm{s}$ to $96 \mathrm{Mbits} / \mathrm{s}$

Li-Fi can offer fast interconnected data systems without causing interference and less power consumption for power plants that need its core temperature, demand, and grid integrity monitoring at all the time. Internet connectivity in tunnels and subway stations poses no obstruction for $\mathrm{Li}-\mathrm{Fi}$ and can extend its powerful means of communication in areas of earthquake and hurricanes disaster. Other possible applications of Li-Fi are smart lighting, intelligent transportation systems, indoor positioning, Internet of Things (IoT), Information 
on prices in artworks collection and listening to streaming auctions of people's work in Museum [6].

Table 1 lists the essential difference between Li-Fi and Wi-Fi technologies.

Table 1 Difference between Li-Fi and Wi-Fi

\begin{tabular}{lll}
\hline Parameters & Li-Fi & Wi-Fi \\
\hline Transmitter & LED & Antenna \\
Receiver & Photodiode & Antenna \\
Interference & Interference is less & Interference is high \\
Privacy and Security & Secure & Not secure \\
Coverage Distance & 10 meters & 32 meters \\
Data Density & High dense environment & Less dense environment \\
Data transfer speed & About 1Gbps & 150Mbps \\
Congestion & Less & More \\
Safety & Safe & Dangerous in hazardous operations \\
Technology & IrDA compliant devices & WLAN 802.11a/b/g/n standard \\
& & compliant devices \\
Frequency of Operation & 10thousand times & 2.4GHz,4.9GHz, and $5 \mathrm{GHz}$ \\
& frequency of Radio & \\
& spectrum & \\
Operation & Using the light with the & Using radio waves with the help of \\
& help of LED bulbs & a Wi-Fi router \\
\hline
\end{tabular}

\section{MATERIALS AND METHOD}

\section{Wi-Fi Simulation}

The performance of the Wi-Fi system when many devices connect to the network was simulated using OPNET Modeler 14.5. The process was carried out by creating a Wi-Fi project with an office network subnet in the OPNET environment. One access point (labelled as wlan_ethernet_slip_4 adv fixed mode), gateway (identified as Ethernet_4 slip_8gateway fixed node), HTTP server (marked as ethernet_server fixed node), ten mobile wireless nodes (marked as WLAN workstation adv mobile node), application configuration and profile configuration were obtained from the object palette tree and placed in the workplace.

The access point connects to the gateway, and then the gateway was connected to the server, which provides applications for the mobile wireless node using an Ethernet 100baseT duplex link. Next, editing of the attributes of the application configuration, profile configuration, server, gateway, access point, and mobile nodes are edited, and their required parameters set for the proper functionality of the system.

For the application configuration parameters, the application name used was the HTTP app, and its description was changed to heavy browsing with a data size of $29.12 \mathrm{MB}$ while the profile configuration name used was pro 1, and its application name changed to HTTP app. The Server's application supported profiles and application supported services were set as pro1 and All respectively. Access point wireless LAN parameters were configured as Basic Service Set (BSS) Identifier: 1, Access Point Functionality: Enabled, Physical Characteristics: Direct Sequence, Data Rate: $11 \mathrm{Mbs}$, Channel Settings: Auto-assigned and Transmit Power: $0.005 \mathrm{~W}$. The same Wireless LAN parameters and attributes set in Access point and Server distinctively was done in the wireless mobile nodes. Instead of changing each of the wireless mobile node's attributes, one of the nodes was selected, right-clicked, and 
then select similar nodes was chosen. With that, all the ten nodes got highlighted, and any of the parameters changed applies to all of them. With all the parameters set in place, the discrete event simulations such as throughput, delay, packet loss, and retransmission and bit error rate were selected, after which the simulation takes place.

\section{Li-Fi Simulation}

Li-Fi network simulation was carried out in Simulink. The proposed system block diagram for Light fidelity simulation is in fig 1

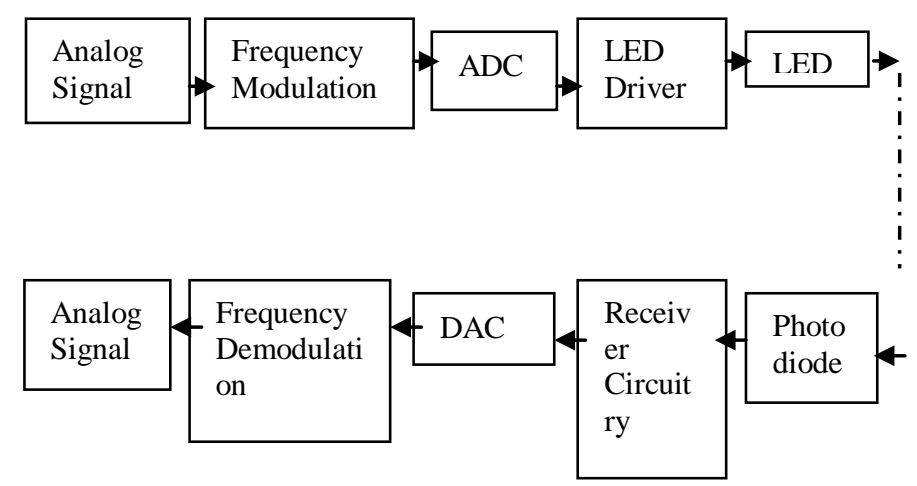

Fig. 1 Simulation Block Diagram of Li-Fi System

Here an analog signal was modulated, transformed into byte format and converted into light signals which are emitted by the LED in the Simulink environment. At the receiver module, the photodiode receives the light signal. The generated binary sequence of the received signal was decrypted to convert data into the original format.

An angle modulated signal has the form

$$
S(t)=A_{c} \cos \left(2 \pi f_{c} t+\theta_{(t)}\right)
$$

Where $A_{c}$ is the amplitude, $f_{c}$ is carrier frequency, $\mathrm{t}$ is the time and $\theta_{(t)}$ is the angle of the carrier signal.

The angle deviation is related to the baseband message $m_{(t)}$ by the expression

$$
\frac{d \theta_{(t)}}{d t}=k_{f} m_{(t)}
$$

where $k_{f}$ is the frequency deviation constant

Integrating (2) gives

$$
\theta_{t}=k_{f} \int_{-\alpha}^{t} m_{\lambda} d \lambda
$$

Substituting (3) into (2) yields

$$
S(t)=A_{c} \cos \left(2 \pi f_{c} t+k_{f} \int_{-\alpha}^{t} m_{\lambda} d \lambda\right)
$$

where $m_{\lambda}$ is the message signal

Simplification of (4) gives

$$
S(t)=A_{c} \cos \left[2 \pi \int_{-\alpha}^{t}\left(f_{c} t+\frac{k_{f}}{2 \pi} m_{\lambda} d \lambda\right)\right]
$$

where $\frac{k_{f}}{2 \pi}$ is the modulation index

Equation (5) was implemented in Simulink environment as shown in figure 2.

A video signal connects to a modulation index whose function was to determine the strength and quality of the transmitted signal. The higher the degree of modulation, the clearer the signal during the reception. An adder block was used to connect the carrier frequency with 
the modulation index of which its output connects to an Integrator. The integrator accumulates the input quantity over a defined time and produces a representative output. The integrator's output connects to a multiplier which does multiplication of the values of integrator output with a gain of $2 \pi$. Its output was connected to a cosine trigonometric function block because cosine term is the real part of a complex exponential and physical measurements can only directly measure real quantities. Then, the output from the cosine trigonometric function block connects to an amplitude carrier $\left(A_{c}\right)$.

The output of the analog frequency modulated signal connects to the $12 \mathrm{bit}$ analog to digital converter that converts the signal to discrete form using the following equation

$$
d_{o}=\frac{2^{n} \times A_{v}}{v_{r e f}}
$$

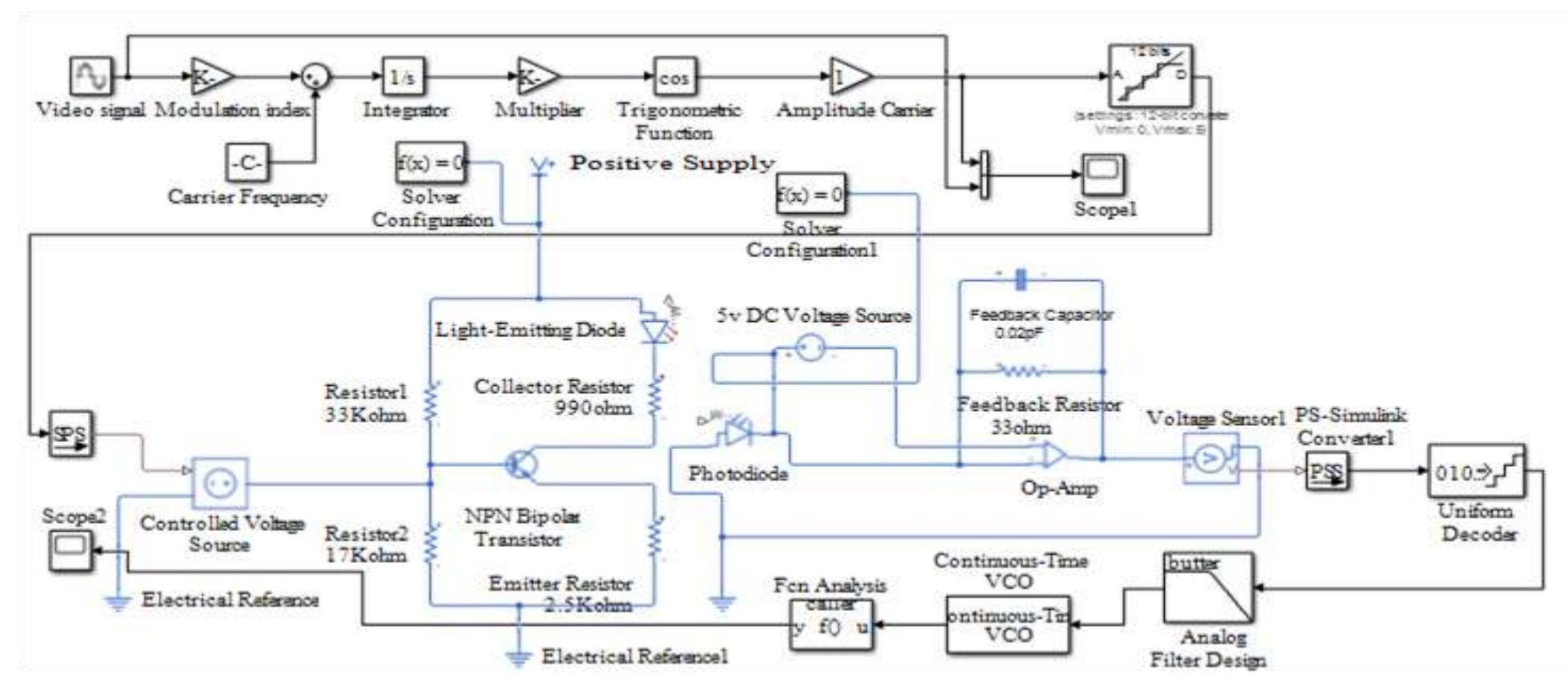

Fig. 2 Simulink model of light fidelity

Where $D_{o}$ is the Digital output, $\mathrm{n}$ is the number of bits in ADC converter, $A_{v}$ is the Analog input voltage and $v_{\text {ref }}$ is the voltage reference of the ADC converter. An Additive White Gaussian Noise (AWGN) was added to the output of the 12 bits analog to digital converter (quantizer). The reason was to make the underlying quantization error relatively more visible. Reamplification was done by connecting the output of AWGN to an amplifier which boosted the signal, spotted noise data, and raised the level of the noise. With these, the signal distinguishes itself from the noise. After that, the signal was pulse shaped using a raised cosine window for filtering, minimizing intersymbol interference, removal of edges and smoothening of the signal. The output signal is fed into the Simulink PS converter which converts it to a physical signal that is injected into a controlled voltage source for an equivalent voltage source. The controlled voltage source connects to a Transistor(NPN) whose function is to control the flow of power to the LED. Though the voltage sensor was connected to the NPN transistor to measure the voltage and convert it to Physical signals from where the PS Simulink converter will convert it to Simulink signals and the output can be viewed through the scope. The Simulink block parameters used for modeling the transmitter are in table 2 . 
In the receiving section, a DC voltage source connects to the photodiode. The photodiode was also connected to the Op-Amp to amplify the current and aid in the conversion of photodiode current to a measurable voltage. The Op-Amp is a transimpedance amplifier.

Table 2 Simulink Transmitter Parameters

\begin{tabular}{|c|c|}
\hline \multirow{3}{*}{$\begin{array}{l}\text { Block Name } \\
\text { Video Signal }\end{array}$} & Parameters \\
\hline & $\begin{array}{l}\text { Sine type based,Time : Use Simulation time,Amplitude:1,Bias and } \\
\text { phase :0 }\end{array}$ \\
\hline & Frequency $: 2 * \mathrm{pi}^{*} 10^{\wedge} 6 \mathrm{~Hz}$, Sample time: $0.001 \mathrm{~s}$, Data Size: $24.12 \mathrm{MB}$ \\
\hline Modulation Index & 100 \\
\hline Carrier Frequency & $100 \mathrm{~Hz}$ \\
\hline Multiplier & $2 *$ pi \\
\hline $\begin{array}{l}\text { Trigonometric } \\
\text { function }\end{array}$ & $\operatorname{Cos}$ \\
\hline Gain & 100 \\
\hline Idealized & Number of converter bits(n):12,Minimum Voltage:0,Maximum \\
\hline Quantizer & Voltage:5 \\
\hline & Output data type: double \\
\hline Simulink-PS & Input Signal Unit: V, Filtering and Derivative: Filter Input, Input \\
\hline Converter & Filtering order: First order filtering, Input filtering time:0.001 sec \\
\hline Resistor & Resistor $=155 \Omega$, Resistor $1=240 \Omega$, Resistor $2=12 \Omega$ \\
\hline NPN Transistor & $\begin{array}{l}\text { Forward current transfer ratio } h_{f e}=100 \text {, Voltage } V_{b e}=0.55 \mathrm{v} \\
\text { Collector Current }=1 \mathrm{~mA} \text {, Collector emitter voltage }=5 \mathrm{~V} \\
\text { Current } I_{b} \text { for Voltage } V_{b e}=0,5 \mathrm{Ma} \text {, Reverse current transfer ratio=1, } \\
\text { Measurement temperature }=25^{\circ} \mathrm{C}\end{array}$ \\
\hline $\begin{array}{l}\text { PS-Simulink } \\
\text { Converter }\end{array}$ & Output Signal Unit=1 \\
\hline $\begin{array}{l}\text { Light } \quad \text { Emitting } \\
\text { Diode }\end{array}$ & Current:0.7A,Voltage $: 5 \mathrm{v}$,Forward Voltage $V_{f}=1.8 \mathrm{v}$ \\
\hline AWGN Channel & $\begin{array}{l}\text { Input processing: frame based,Initial seed:67, Mode : Signal to noise } \\
\text { ratio(Eb/No),Eb/No (dB):20,No of bits per symbol: } 16 \text {,Input signal } \\
\text { power,referenced to } 1 \mathrm{ohm} \text { (watts) : } 1 \text {,Symbol period: } 1\end{array}$ \\
\hline Amplifier & $\begin{array}{l}\text { Method: Linear.Linear gain }(\mathrm{dB}): 3 \text {,Specification method: Noise } \\
\text { factor,Noise factor:2,Initial Seed:67 }\end{array}$ \\
\hline Raised Cosine & Filter shape: Square root,Roll-off factor : 0.2,Filter span in \\
\hline Transmit Filter & $\begin{array}{l}\text { symbols):10, Output samples per symbol: } 8 \text {, Linear amplitude filter } \\
\text { gain :1,Input processing: frame-based, Rate options: Enforce single } \\
\text { rate processing }\end{array}$ \\
\hline
\end{tabular}

Its output was connected to the voltage sensor to measure the voltage signal and sends the output to the PS-Simulink Converter. The idea is to convert the Physical signal to Simulink equivalent signal since the next bock which needed to connect with it is not a Simscape block. The output of PS-Simulink Converter attaches to the uniform decoder whose function is to recover the signal back to an analog signal. Next, the analog filter was connected to the uniform decoder to pass high frequencies, and its output was connected to the continuoustime voltage controlled oscillator to produce an output signal of a variable frequency that is directly proportional to the magnitude of the input voltage. The output connects to the $\mathrm{Fcn}$ analysis block, which was configured to represent latency, packet loss, BER, Speed, and throughput. The parameters used for the receiver section is as presented in table 3 . 
Table 3 Simulink Receiver Parameters

\begin{tabular}{|c|c|}
\hline Block Name & Parameters \\
\hline Photodiode & $\begin{array}{l}\text { Current }=25 \mu \mathrm{A} \\
\text { Flux density }=5 \mathrm{w} / \mathrm{m}^{2} \\
\text { Current } I_{f} \text { at } V_{f}=0.1 \mathrm{~A} \\
\text { Forward voltage } V_{f}=1.3 \mathrm{v} \\
\text { Dark Current }=5 \times 10^{-9} \mathrm{~A} \\
\text { Measurement Temperature }=25^{\circ} \mathrm{C}\end{array}$ \\
\hline Voltage Source & $12 \mathrm{v}$ \\
\hline Operational Amplifier (Op-Amp) & $\begin{array}{l}\text { Current }=3.3 \mu \mathrm{A} \\
\text { Resistor } 3=100 \mathrm{~K} \Omega\end{array}$ \\
\hline Uniform Decoder & $\begin{array}{l}\text { Bits }=8 \\
\text { Peak }=1 \\
\text { Output type=Double }\end{array}$ \\
\hline Analog Filter Design & $\begin{array}{l}\text { Design method: Butterworth } \\
\text { Filter type: High pass } \\
\text { Filter order: } 8 \\
\text { Passband edge frequency: } 2 * \mathrm{pi}^{*} 1000000 \mathrm{rad} / \mathrm{sec}\end{array}$ \\
\hline Continuous time VCO & $\begin{array}{l}\text { Output amplitude: } 1 \mathrm{v} \\
\text { Input sensitivity: } 1 \\
\text { Initial phase }(\mathrm{rad})=0 \\
\text { Quiescent frequency }=10 \mathrm{MHz}\end{array}$ \\
\hline Fcn Analysis & $\begin{array}{l}\text { Throughput, Latency, Packet loss, BER, Data } \\
\text { Speed }\end{array}$ \\
\hline AWGN Channel & $\begin{array}{l}\text { Input processing: frame-based } \\
\text { Initial seed: } 67 \\
\text { Mode: Signal to noise ratio(Eb/No) } \\
\text { Eb/No (dB): } 20 \\
\text { No of bits per symbol: } 16 \\
\text { Input signal power,referenced to } 1 \mathrm{ohm}(\text { watts) }: 1 \\
\text { Symbol period: } 1\end{array}$ \\
\hline Amplifier & $\begin{array}{l}\text { Method: Linear } \\
\text { Linear gain }(\mathrm{dB}): 3 \\
\text { Specification method: Noise factor } \\
\text { Noise factor:2 } \\
\text { Initial Seed: } 67\end{array}$ \\
\hline Raised Cosine Transmit Filter & $\begin{array}{l}\text { Filter shape: Square root } \\
\text { Roll-off factor: } 0.2 \\
\text { Filter span in symbols): } 10 \\
\text { Output samples per symbol: } 8 \\
\text { Linear amplitude filter gain: } 1 \\
\text { Input processing: frame-based } \\
\text { Rate options: Enforce single rate processing }\end{array}$ \\
\hline
\end{tabular}




\section{RESULTS}

The result obtained from the simulation of Wi-Fi is in fig 3
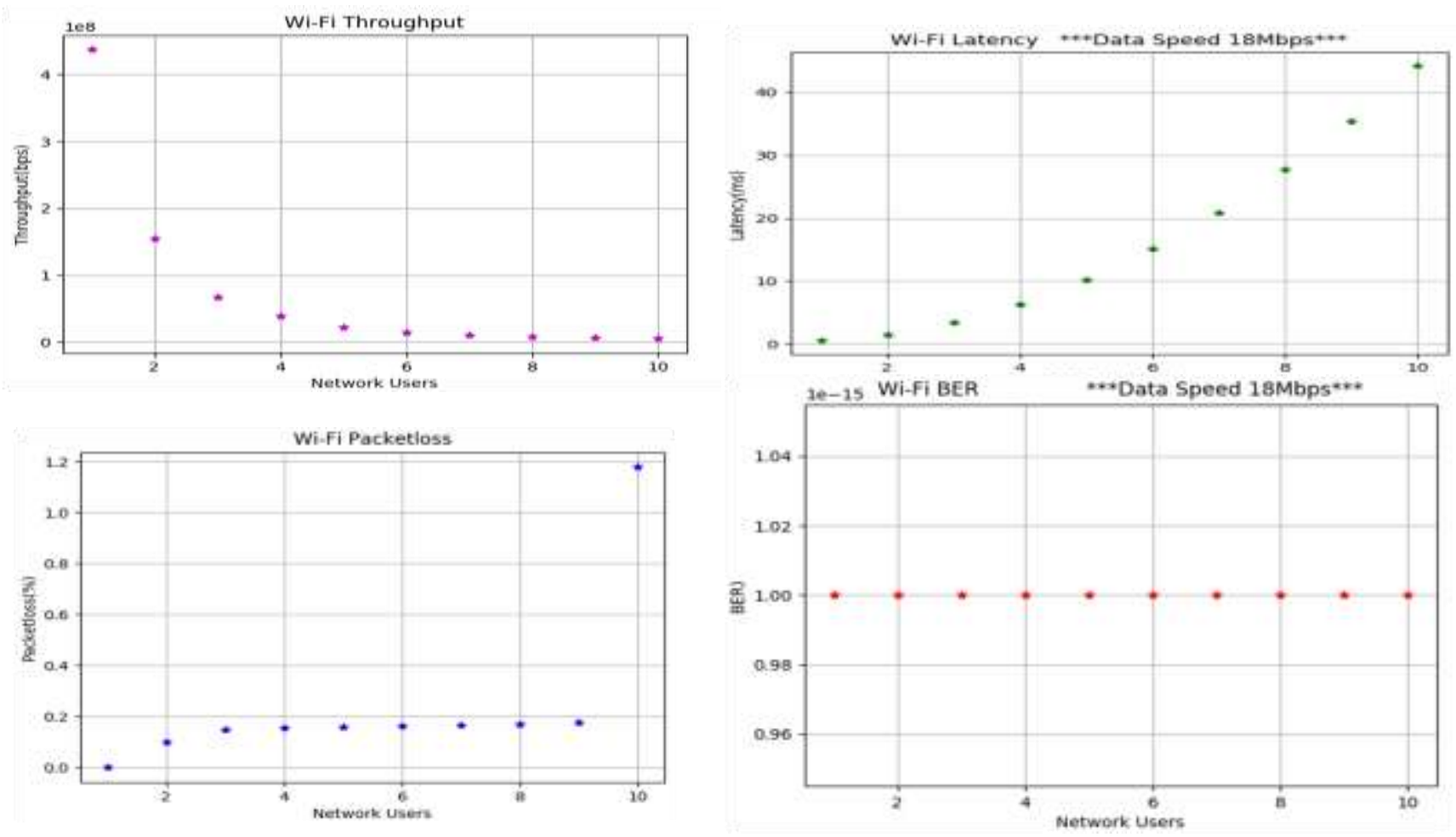

Fig. 3 Wireless fidelity simulation

The result in fig. 3 showed that the throughput decreases as more users connect to the Wi-Fi System. It was because the users shared the available bandwidth. It can be seen to have an exponential decay. Between the fourth user and tenth user, the decrease tapers. The tenth user was tending to zero. Thus by extrapolation, it can be seen that connecting more than users will result in zero output. The figure also showed that the latency had exponential growth. The reason behind it is that increasing the number of users will increase transmission time delay of all packet sizes. Packet loss increased from the first to the third user and maintained a constant value. After the ninth user, it rose exponentially. As more users are downloading data, several packets are lost. The bit error rate was high and the same all through the measurement. It showed that it does not depend significantly on the number of users.

Li-Fi Simulation result is in fig 4. From the result, there was high throughput, low latency, low packet loss, and an acceptable BER value of $10^{-10}$. The data throughput was steady when the fourth user was connected. It dropped a little bit when the fifth user was connected but maintained the same value from eighth to the tenth user. Also, the trend of decreasing and maintaining the same value is in latency and packet loss. The network performance of the LiFi system is not significantly affected by the number of users. Observation showed that the data speed was $27 \mathrm{Mbps}$, which is better than the speed of Wi-Fi (18Mbps).

The performance results of the comparison of the throughput, latency, packet loss, and BER values with the simulated Wi-Fi network system using the Python program is in fig 5

The result in fig. 5 showed that the performance of data throughput and other network performance parameters such as Latency, Packet Loss, and BER are better in the Li-Fi system than Wi-Fi. Data throughput in the Li-Fi system is not significantly affected when multiple 
users connect in the network, unlike in Wi-Fi and as such will find massive applicability in a highly dense area. It has shown that data transfer using the Li-Fi system is better than the WiFi system. The percentage decrease in the data throughput of Wi-Fi was calculated and found to be $98.8 \%$ while the percentage decrease in the data throughput of Li-Fi was $45.8 \%$. Hence the percentage improvement of the data throughput of $\mathrm{Li}-\mathrm{Fi}$ against $\mathrm{Wi}-\mathrm{Fi}$ is $53 \%$. The percentage increase in the latency and packet loss of Wi-Fi was calculated and found to be $68.8 \%$ and $10.78 \%$ while that of $\mathrm{Li}-\mathrm{Fi}$ was $29.7 \%$ and $2 \%$ respectively
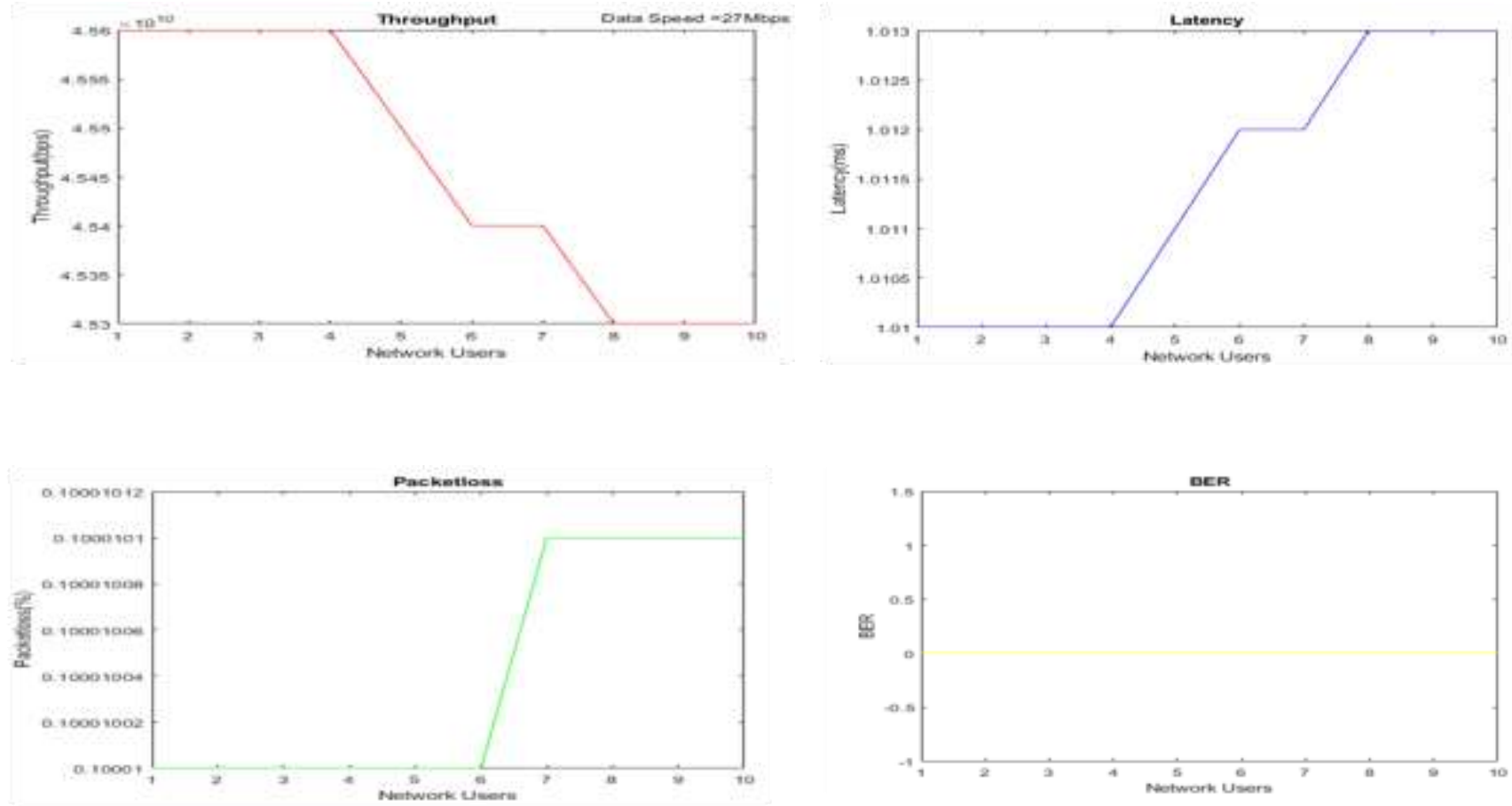

Fig. 4 Result of light fidelity simulation
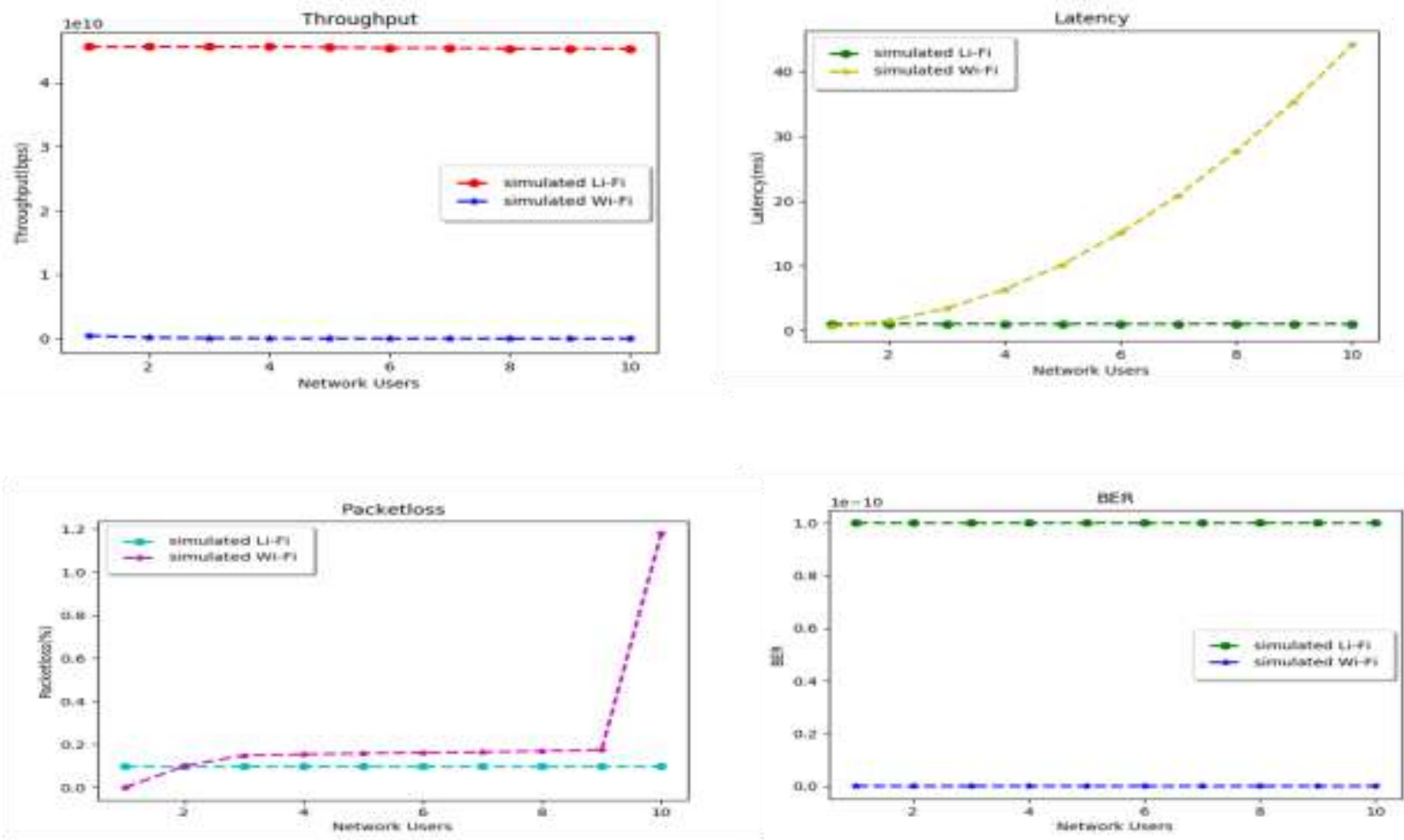

Fig. 5 Network performance comparison of Li-Fi and Wi-Fi Network 


\section{CONCLUSION}

$\mathrm{Li}-\mathrm{Fi}$ is an optical version of Wi-Fi and uses a transceiver fitted with LED lamps instead of Wi-Fi modems. It is a promising technology that is currently attracting a great deal of interest because of its speed. Light Fidelity is green communication technology. It saves energy, reuses the existing lighting infrastructure, and thereby reduces $\mathrm{CO}_{2}$ emission. Li-Fi can lead to the internet of things (IoT) if the LED lights on the electronics have internet access. It is the answer to our digital future where the lights in the room, street, everywhere will be used for transmission of data to laptops, smartphones, and tablets.

\section{REFERENCES}

[1] Pushpendra, V., Jayant, S., Preety, Asthana, A. (2015) Light Fidelity: Transmission of Data through Light of Future Technology. International Journal of Computer Sciences and Mobile Computing, ISSN: 2320 - 088X, 4(9), 113-124.

[2] Haas H. (2015). What is Li-Fi?. Journal of Lightwave Technology, 34( 6), 1533-1544.

[3] Prateek, G., Aditya, S., Prashank, K. (2016). Introduction to Wi-Fi Wireless Networking. International Journal of Advanced Research in Computers and Communication Engineering(IJARCCE), 5( 3), 121-125.

[4] Bradley, K. (2016). Introduction to Wi-Fi Wireless Networking. LifeWire. https://www.lifewire.com/introduction-to-wi-fi-wireless-networking-818265. Accessed on August 2018.

[5] Abhishek, P., Akash, A., Arpit, B., Indrago, K., (2015). LiFi: Data Transmission through Illumination. International Journal of Scientific and Engineering Research, 6(10), 14321436.

[6] Iyidiobi, J. C., Arinze, S.N., Okafor, P. U. (2018). Light Fidelity: The Future to the Wireless Data Communication” American Journal of Engineering Research, 7(10),.245-248.

[7] Lathi, B. P., Ding, Z. (2009). Modern Digital and Analog Communication. The Oxford Series in Electrical and Computer Engineering, fourth edition, ISBN:9780195384932, pp.1928. 